\title{
A new class of unified integral formulas associated with whittaker functions
}

\author{
N. U. Khan, T. Usman and M. Ghayasuddin
}

Department of Applied Mathematics, Aligarh Muslim University, Aligarh, India

Received: 21 August 2015, Revised: 30 October 2015, Accepted: 18 December 2015

Published online: 10 February 2016.

\begin{abstract}
In recent years, integral formulas involving a variety of special functions have been developed by a number of authors. Recently Khan and Ghayasuddin established some interesting unified integrals involving Whittaker function, generalized Bessel function and generalized Bessel-Maitland functions. In the present paper, we aim at establishing two new generalized integral formulas involving Whittaker function of first kind $M_{k, \mu}(z)$, which are expressed in terms of Kampé de Fériet functions. Our integrals are unified in nature and act as key formulas from which we also derived some special cases.
\end{abstract}

Keywords: Whittaker function, Kampé de Fériet functions and Integrals.

\section{Introduction}

Many important functions in applied sciences are defined via improper integrals or series (or infinite products). The general name of these important functions are called special functions. Bessel function are important special functions and their closely related ones are widely used in physics and engineering; therefore, they are of interest to physicists and engineers as well as mathematicians. In recent years, numerous integral formulas involving a variety of special functions have been developed by many authors (see, [1], [2], [3], [4], [6] and [9]). Recently Khan and Ghayasuddin [8] established some interesting unified integrals involving the Whittaker function of first kind $M_{\rho, \sigma}(z)$. Afterwards Khan and Ghayasuddin [10] defined some another new integral formulas involving generalized Bessel function $w_{v}(z)$, in terms of Wright hypergeometric function. Motivated by the above mentioned work, we present two another new unified integrals involving the Whittaker function of first kind $M_{k, \mu}(z)$, which are expressed in terms of the Kampé de Fériet functions.

The Whittaker functions $M_{k, \mu}(z)$ and $W_{k, \mu}(z)$ were introduced by Whittaker [12] (see also Whittaker and Watson [13]) in terms of confluent hypergeometric function ${ }_{1} F_{1}$ (or Kummer's functions):

$$
M_{k, \mu}(z)=z^{\mu+\frac{1}{2}} e^{-z / 2}{ }_{1} F_{1}\left(\frac{1}{2}+\mu-k ; 2 \mu+1 ; z\right) .
$$

and

$$
W_{k, \mu}(z)=z^{\mu+\frac{1}{2}} e^{-z / 2} U\left(\frac{1}{2}+\mu-k ; 2 \mu+1 ; z\right)
$$

However the confluent hypergeometric function disappears when $2 \mu$ is an integer, so whittaker functions are often defined instead. The whittaker functions are related to the parabolic cylinder functions. 
When $|\arg (z)|<\frac{3 \pi}{2}$ and $2 \mu$ is not an integer,

$$
W_{k, \mu}(z)=\frac{\Gamma(-2 \mu)}{\Gamma\left(\frac{1}{2}-\mu-k\right)} M_{k, \mu(z)}+\frac{\Gamma(2 \mu)}{\Gamma\left(\frac{1}{2}+\mu-k\right)} M_{k,-\mu}(z)
$$

When $|\arg (-z)|<\frac{3 \pi}{2}$ and $2 \mu$ is not an integer,

$$
W_{-k, \mu}(z)=\frac{\Gamma(-2 \mu)}{\Gamma\left(\frac{1}{2}-\mu-k\right)} M_{-k, \mu(-z)}+\frac{\Gamma(2 \mu)}{\Gamma\left(\frac{1}{2}+\mu+k\right)} M_{-k,-\mu}(-z) .
$$

In 1921, the four Appell functions were unified and generalized by Kampé de Fériet, who defined a general hypergeometric function of two variables. The notation introduced by Kampé de Fériet for his double hypergeometric function of superior order was subsequently abbreviated by Burchnall and Chaundy. We recall here the definition of a more general double hypergeometric function in a slightly modified notation (see [11]):

$$
F_{l: m ; n}^{p: q ; k}\left[\begin{array}{l}
\left(a_{p}\right):\left(b_{q}\right) ;\left(c_{k}\right) ; \\
\left(\alpha_{l}\right):\left(\beta_{m}\right) ;\left(\gamma_{n}\right) ;
\end{array} x, y\right]=\sum_{r, s=0}^{\infty} \frac{\prod_{j=1}^{p}\left(a_{j}\right)_{r+s} \prod_{j=1}^{q}\left(b_{j}\right)_{r} \prod_{j=1}^{k}\left(c_{j}\right)_{s}}{\prod_{j=1}^{l}\left(\alpha_{j}\right)_{r+s} \prod_{j=1}^{m}\left(\beta_{j}\right)_{r} \prod_{j=1}^{n}\left(\gamma_{j}\right)_{s}} \frac{x^{r}}{r !} \frac{y^{s}}{s !}
$$

where, for convergence,

(i) $p+q<l+m+1, \quad p+k<l+n+1, \quad|x|<\infty, \quad|y|<\infty$, or

(ii) $p+q=l+m+1, \quad p+k=l+n+1$, and

$$
\left\{\begin{array}{cc}
|x|^{1 /(p-l)}+|y|^{1 /(p-l)}<1, & \text { if } p>l, \\
\max \{|x|,|y|\}<1, & \text { if } p \leq l .
\end{array}\right.
$$

For our present investigation, the following interesting and useful result due to Lavoie and Trottier [7] will be required

$$
\int_{0}^{1} x^{\alpha-1}(1-x)^{2 \beta-1}\left(1-\frac{x}{3}\right)^{2 \alpha-1}\left(1-\frac{x}{4}\right)^{\beta-1} d x=\left(\frac{2}{3}\right)^{2 \alpha} \frac{\Gamma(\alpha) \Gamma(\beta)}{\Gamma(\alpha+\beta)},(\Re(\alpha)>0 \text { and } \Re(\beta)>0) .
$$

\section{Main results}

In this section, we establish two generalized integral formulas involving the Whittaker function, which are expressed in terms of Kampé de Fériet functions.

First Integral: The following integral formula holds true: for $\Re(\alpha)>0, \Re(\beta+\mu)>-\frac{1}{2}$,

$$
\begin{gathered}
\int_{0}^{1} x^{\alpha-1}(1-x)^{2 \beta-1}\left(1-\frac{x}{3}\right)^{2 \alpha-1}\left(1-\frac{x}{4}\right)^{\beta-1} M_{k, \mu}\left[y\left(1-\frac{x}{4}\right)(1-x)^{2}\right] d x=\left(\frac{2}{3}\right)^{2 \alpha} y^{\mu+\frac{1}{2}} \\
\left.\times \frac{\Gamma(\alpha) \Gamma\left(\beta+\mu+\frac{1}{2}\right)}{\Gamma\left(\alpha+\beta+\mu+\frac{1}{2}\right)} F_{1: 1 ; 0}^{1: 1: 0}\left[\begin{array}{c}
\beta+\mu+\frac{1}{2}: \quad \mu-k+\frac{1}{2} ; \ldots ; \\
\alpha+\beta+\mu+\frac{1}{2}: 2 \mu+1 ; \ldots
\end{array}\right],-\frac{y}{2}\right] .
\end{gathered}
$$


Second Integral: The following integral formula holds true: for $\Re(\alpha+\mu)>-\frac{1}{2}, \Re(\beta+\mu)>-\frac{1}{2}$,

$$
\begin{aligned}
& \int_{0}^{1} x^{\alpha-1}(1-x)^{2 \beta-1}\left(1-\frac{x}{3}\right)^{2 \alpha-1}\left(1-\frac{x}{4}\right)^{\beta-1} M_{k, \mu}\left[x y\left(1-\frac{x}{4}\right)(1-x)^{2}\right] d x=\left(\frac{2}{3}\right)^{2 \alpha} y^{\mu+\frac{1}{2}} \\
& \times \frac{\Gamma\left(\alpha+\mu+\frac{1}{2}\right) \Gamma\left(\beta+\mu+\frac{1}{2}\right)}{\Gamma(\alpha+\beta+2 \mu+1)} F_{2: 1 ; 0}^{2: 1: 0}\left[\begin{array}{l}
\left.\alpha+\mu+\frac{1}{2}, \beta+\mu+\frac{1}{2}: \mu-k+\frac{1}{2} ;-\frac{y}{4},-\frac{y}{8}\right] . \\
\frac{\alpha+\beta+2 \mu+1}{2}, \frac{\alpha+\beta+2 \mu+2}{2}: 2 \mu+1 ; \ldots
\end{array}\right]
\end{aligned}
$$

Proof. In order to derive our first integral (8), we proceed as follows. Denoting the left-hand side of (8) by I, expressing $M_{k, \mu}$ as a series with the help of (1), changing the order of integration and summation (which is verified by uniform convergence of the involved series under the given conditions), we get

$$
\begin{gathered}
\mathrm{I}=\mathrm{y}^{\mu+\frac{1}{2}} \sum_{\mathrm{r}, \mathrm{n}=0}^{\infty} \frac{\left(\mu-\mathrm{k}+\frac{1}{2}\right)_{\mathrm{n}}}{(2 \mu+1)_{\mathrm{n}}} \frac{(\mathrm{y})^{\mathrm{n}}}{\mathrm{n} !} \frac{\left(-\frac{\mathrm{y}}{2}\right)^{\mathrm{r}}}{\mathrm{r} !} \\
\times \int_{0}^{1} x^{\alpha-1}(1-x)^{2 \beta+2 \mu+2 r+2 n}\left(1-\frac{x}{3}\right)^{2 \alpha-1}\left(1-\frac{x}{4}\right)^{\beta+\mu+r+n-\frac{1}{2}} d x .
\end{gathered}
$$

Evaluating the above integral with the help of (1.6) and using the result $(a)_{n}=\frac{\Gamma(a+n)}{\Gamma(a)}$, we get

$$
\mathrm{I}=\left(\frac{2}{3}\right)^{2 \alpha} \mathrm{y}^{\mu+\frac{1}{2}} \frac{\Gamma(\alpha) \Gamma\left(\beta+\mu+\frac{1}{2}\right)}{\Gamma\left(\alpha+\beta+\mu+\frac{1}{2}\right)} \sum_{\mathrm{r}, \mathrm{n}=0}^{\infty} \frac{\left(\beta+\mu+\frac{1}{2}\right)_{\mathrm{r}+\mathrm{n}}\left(\mu-\mathrm{k}+\frac{1}{2}\right)_{\mathrm{n}}}{\left(\alpha+\beta+\mu+\frac{1}{2}\right)_{\mathrm{r}+\mathrm{n}}(2 \mu+1)_{\mathrm{n}}} \frac{\mathrm{y}^{\mathrm{n}}}{\mathrm{n} !} \frac{\left(-\frac{\mathrm{y}}{2}\right)^{\mathrm{r}}}{\mathrm{r} !}
$$

Finally, summing up the above series with the help of the definition (5), we arrive at the right-hand side of (8). This completes the proof of our first result.

Proof. Similarly, to derive our second integral (9), denoting the left-hand side of (9) by I'. On expressing $M_{k, \mu}$ as a series with the help of (1), changing the order of integration and summation (which is verified by uniform convergence of the involved series under the given conditions), we get

$$
\begin{gathered}
\mathrm{I}^{\prime}=(\mathrm{y})^{\mu+\frac{1}{2}} \sum_{\mathrm{r}, \mathrm{n}=0}^{\infty} \frac{\left(\mu-\mathrm{k}+\frac{1}{2}\right)_{\mathrm{n}}}{(2 \mu+1)_{\mathrm{n}}} \frac{(\mathrm{y})^{\mathrm{n}}}{\mathrm{n} !} \frac{\left(-\frac{\mathrm{y}}{2}\right)^{\mathrm{r}}}{\mathrm{r} !} \\
\times \int_{0}^{1} x^{\alpha+\mu+r+n+\frac{1}{2}}(1-x)^{2 \beta+2 \mu+2 r+2 n}\left(1-\frac{x}{3}\right)^{2 \alpha-1}\left(1-\frac{x}{4}\right)^{\beta+\mu+r+n-\frac{1}{2}} d x .
\end{gathered}
$$

Evaluating the above integral with the help of (7) and using the following results;

$$
(a)_{n}=\frac{\Gamma(a+n)}{\Gamma(a)} \text { and }(\lambda)_{2 n}=2^{2 n}\left(\frac{\lambda}{2}\right)_{n}\left(\frac{\lambda+1}{2}\right)_{n},
$$

where $(a)_{n}$ is called the Pochhammer's symbol [5] and after a little simplification, we have

$$
\begin{gathered}
\mathrm{I}^{\prime}=\left(\frac{2}{3}\right)^{2 \alpha}(\mathrm{y})^{\mu+\frac{1}{2}} \frac{\Gamma\left(\alpha+\mu+\frac{1}{2}\right) \Gamma\left(\beta+\mu+\frac{1}{2}\right)}{\Gamma(\alpha+\beta+2 \mu+1)} \\
\times \sum_{r, n=0}^{\infty} \frac{\left(\alpha+\mu+\frac{1}{2}\right)_{r+n}\left(\beta+\mu+\frac{1}{2}\right)_{r+n}\left(\mu-k+\frac{1}{2}\right)_{n}}{\left(\frac{\alpha+\beta+2 \mu+1}{2}\right)_{r+n}\left(\frac{\alpha+\beta+2 \mu+2}{2}\right)_{r+n}(2 \mu+1)_{n}} \frac{\left(-\frac{y}{2}\right)^{r}}{n !} .
\end{gathered}
$$

which, upon using the definition (5), yields (9). This completes the proof of our second result. 


\section{Special cases}

In this section, we derive certain new integral formulas for the exponential functions, Modified Bessel functions, Sine hyperbolic functions, Laguerre polynomials and Hermite polynomials. To do this, we recall here the following known relations:

$$
\begin{gathered}
M_{k,-k-\frac{1}{2}}(z)=e^{\frac{z}{2}} z^{-k} . \\
M_{0, \mu}(2 z)=2^{2 \mu+\frac{1}{2}} \Gamma(1+\mu) \sqrt{z} I_{\mu}(z),
\end{gathered}
$$

where $I_{\mu}(z)$ is Modified Bessel function [11].

$$
\begin{gathered}
M_{0, \frac{1}{2}}(2 z)=2 \sinh z . \\
M_{\frac{p}{2}+\frac{1}{2}+m, \frac{p}{2}}(z)=\frac{m !}{(p+1)_{m}} e^{-\frac{z}{2}} z^{\frac{p}{2}+\frac{1}{2}} L_{m}^{p}(z),
\end{gathered}
$$

where $L_{m}^{p}(z)$ is the generalized Laguerre polynomial [11].

$$
\begin{gathered}
M_{\frac{1}{4}+p,-\frac{1}{4}}\left(z^{2}\right)=(-1)^{p} \frac{p !}{2 p !} e^{\frac{-z^{2}}{2}} \sqrt{z} H_{2 p}(z), \\
M_{\frac{3}{4}+p, \frac{1}{4}}\left(z^{2}\right)=(-1)^{p} \frac{p !}{(2 p+1) !} \frac{e^{\frac{-z^{2}}{2}} \sqrt{z}}{2} H_{2 p+1}(z) .
\end{gathered}
$$

where $H_{p}(z)$ is the generalized Hermite polynomial [11].

(i) On setting $\mu=-k-\frac{1}{2}$ and using (10) in our first integral (8), we get

$$
\begin{aligned}
& \int_{0}^{1} x^{\alpha-1}(1-x)^{2 \beta-2 k-1}\left(1-\frac{x}{3}\right)^{2 \alpha-1}\left(1-\frac{x}{4}\right)^{\beta-k-1} \exp \left[\frac{y\left(1-\frac{x}{4}\right)(1-x)^{2}}{2}\right] d x \\
& =\left(\frac{2}{3}\right)^{2 \alpha} \frac{\Gamma(\alpha) \Gamma(\beta-k)}{\Gamma(\alpha+\beta-k)} F_{1: 0 ; 0}^{1: 0: 0}\left[\begin{array}{c}
\beta-k:-;-; \\
\alpha+\beta-k: \ldots ; \ldots ;
\end{array}\right]
\end{aligned}
$$

where $\mathfrak{R}(\alpha)>0, \mathfrak{R}(\beta-k)>0$.

(ii) Further, on setting $\mu=-k-\frac{1}{2}$ and using (10) in our second integral (9), we get

$$
\begin{aligned}
& \int_{0}^{1} x^{\alpha-k-1}(1-x)^{2 \beta-2 k-1}\left(1-\frac{x}{3}\right)^{2 \alpha-1}\left(1-\frac{x}{4}\right)^{\beta-k-1} \exp \left[\frac{x y\left(1-\frac{x}{4}\right)(1-x)^{2}}{2}\right] d x \\
& =\left(\frac{2}{3}\right)^{2 \alpha} \frac{\Gamma(\alpha-k) \Gamma(\beta-k)}{\Gamma(\alpha+\beta-2 k)} F_{2: 0 ; 0}^{2: 0: 0}\left[\begin{array}{c}
\alpha-k, \quad \beta-k: \quad ; \ldots ; \\
\frac{\alpha+\beta-2 k}{2}, \frac{\alpha+\beta-2 k+1}{2}: \ldots ;
\end{array} ;\right.
\end{aligned}
$$

where $\mathfrak{R}(\alpha-k)>0, \mathfrak{R}(\beta-k)>0$. 
(iii) On setting $k=0$ and using (11) in our first integral (8), we get

$$
\begin{aligned}
& \int_{0}^{1} x^{\alpha-1}(1-x)^{2 \beta}\left(1-\frac{x}{3}\right)^{2 \alpha-1}\left(1-\frac{x}{4}\right)^{\beta-\frac{1}{2}} I_{\mu}\left[\frac{y\left(1-\frac{x}{4}\right)(1-x)^{2}}{2}\right] d x=\left(\frac{1}{3}\right)^{2 \alpha} 2^{2(\alpha-\mu)} \\
& \times \frac{\Gamma(\alpha) \Gamma\left(\beta+\mu+\frac{1}{2}\right)}{\Gamma(1+\mu) \Gamma\left(\alpha+\beta+\mu+\frac{1}{2}\right)} F_{1: 1 ; 0}^{1: 1: 0}\left[\begin{array}{c}
\beta+\mu+\frac{1}{2}: \mu+\frac{1}{2} ;-; \\
\alpha+\beta+\mu+\frac{1}{2}: 2 \mu+1 ;
\end{array} ;,-\frac{y}{2}\right],
\end{aligned}
$$

where $\mathfrak{R}(\alpha)>0, \mathfrak{R}(\beta+\mu)>-\frac{1}{2}$.

(iv) Further, on setting $k=0$ and using (11) in our second integral (9), we get

$$
\begin{aligned}
& \int_{0}^{1} x^{\alpha-\frac{1}{2}}(1-x)^{2 \beta}\left(1-\frac{x}{3}\right)^{2 \alpha-1}\left(1-\frac{x}{4}\right)^{\beta-\frac{1}{2}} I_{\mu}\left[\frac{x y\left(1-\frac{x}{4}\right)(1-x)^{2}}{2}\right] d x=\left(\frac{1}{3}\right)^{2 \alpha} 2^{2(\alpha-\mu)} y^{\mu} \\
& \times \frac{\Gamma\left(\alpha+\mu+\frac{1}{2}\right) \Gamma\left(\beta+\mu+\frac{1}{2}\right)}{\Gamma(1+\mu) \Gamma(\alpha+\beta+2 \mu+1)} F_{2: 1 ; 0}^{2: 1: 0}\left[\begin{array}{l}
\alpha+\mu+\frac{1}{2}, \beta+\mu+\frac{1}{2}: \mu+\frac{1}{2} ;-; \\
\frac{\alpha+\beta+2 \mu+1}{2}, \frac{\alpha+\beta+2 \mu+2}{2}: 2 \mu+1 ; \ldots ;
\end{array}\right]
\end{aligned}
$$

where $\Re(\alpha+\mu)>-\frac{1}{2}, \Re(\beta+\mu)>-\frac{1}{2}$.

(v) On setting $k=0, \mu=\frac{1}{2}$ and using (12) in our first integral (8), we get

$$
\begin{aligned}
& \int_{0}^{1} x^{\alpha-1}(1-x)^{2 \beta-1}\left(1-\frac{x}{3}\right)^{2 \alpha-1}\left(1-\frac{x}{4}\right)^{\beta-1} \sinh \left[\frac{y\left(1-\frac{x}{4}\right)(1-x)^{2}}{2}\right] d x=\left(\frac{1}{3}\right)^{2 \alpha} \\
& \times 2^{2 \alpha-1} y \frac{\Gamma(\alpha) \Gamma(\beta+1)}{\Gamma(\alpha+\beta+1)} F_{1: 1 ; 0}^{1: 1: 0}\left[\begin{array}{cc}
\beta+1: 1 ;-; & \left.;,-\frac{y}{2}\right] \\
\alpha+\beta+1: 2 ; & ;
\end{array}\right]
\end{aligned}
$$

where $\mathfrak{R}(\alpha)>0, \Re(\beta)>0$.

(vi) Further, on setting $k=0, \mu=\frac{1}{2}$ and using (12) in our second integral (9), we get

$$
\begin{aligned}
& \int_{0}^{1} x^{\alpha-1}(1-x)^{2 \beta-1}\left(1-\frac{x}{3}\right)^{2 \alpha-1}\left(1-\frac{x}{4}\right)^{\beta-1} \sinh \left[\frac{x y\left(1-\frac{x}{4}\right)(1-x)^{2}}{2}\right] d x=\left(\frac{1}{3}\right)^{2 \alpha} 2^{2 \alpha-1} \\
& \times y \frac{\Gamma(\alpha+1) \Gamma(\beta+1)}{\Gamma(\alpha+\beta+2)} F_{2: 1 ; 0}^{2: 1: 0}\left[\begin{array}{ccc}
\alpha+1, & \beta+1: 1 ; \ldots ; \\
\frac{\alpha+\beta+2}{2}, & \frac{\alpha+\beta+3}{2}: 2 ; \ldots ;
\end{array}\right],
\end{aligned}
$$

where $\mathfrak{R}(\alpha)>0, \Re(\beta)>0$. 
(vii) On setting $k=\frac{p}{2}+\frac{1}{2}+m$ (m is non negative integer) $\mu=\frac{p}{2}$ and using (13) in our first integral (8), we get

$$
\begin{aligned}
& \int_{0}^{1} x^{\alpha-1}(1-x)^{2 \beta+p}\left(1-\frac{x}{3}\right)^{2 \alpha-1}\left(1-\frac{x}{4}\right)^{\beta+\frac{p}{2}-\frac{1}{2}} \exp \left[\frac{y\left(1-\frac{x}{4}\right)(1-x)^{2}}{2}\right] \\
& \times L_{m}^{p}\left[y\left(1-\frac{x}{4}\right)(1-x)^{2}\right] d x=\left(\frac{2}{3}\right)^{2 \alpha} \frac{(p+1)_{m}}{m !} \frac{\Gamma(\alpha) \Gamma\left(\beta+\frac{p}{2}+\frac{1}{2}\right)}{\Gamma\left(\alpha+\beta+\frac{p}{2}+\frac{1}{2}\right)} \\
& \times F_{1: 1 ; 0}^{1: 1: 0}\left[\begin{array}{c}
\beta+\frac{p}{2}+\frac{1}{2}:-m ; \ldots ; \\
\alpha+\beta+\frac{p}{2}+\frac{1}{2}: p+1 ;
\end{array} \quad y,-\frac{y}{2}\right],
\end{aligned}
$$

where $\mathfrak{R}(\alpha)>0, \mathfrak{R}\left(\beta+\frac{p}{2}\right)>-\frac{1}{2}$.

(viii) Further, on setting $k=\frac{p}{2}+\frac{1}{2}+m$ ( $\mathrm{m}$ is non negative integer) $\mu=\frac{p}{2}$ and using (13) in our second integral (9), we get

$$
\begin{aligned}
& \int_{0}^{1} x^{\alpha+\frac{p}{2}+\frac{1}{2}}(1-x)^{2 \beta+p}\left(1-\frac{x}{3}\right)^{2 \alpha-1}\left(1-\frac{x}{4}\right)^{\beta+\frac{p}{2}-\frac{1}{2}} \exp \left[\frac{x y\left(1-\frac{x}{4}\right)(1-x)^{2}}{2}\right] \\
& \times L_{m}^{p}\left[x y\left(1-\frac{x}{4}\right)(1-x)^{2}\right] d x=\left(\frac{2}{3}\right)^{2 \alpha} \frac{(p+1)_{m}}{m !} \frac{\Gamma\left(\alpha+\frac{p}{2}+\frac{1}{2}\right) \Gamma\left(\beta+\frac{p}{2}+\frac{1}{2}\right)}{\Gamma(\alpha+\beta+p+1)} \\
& \times F_{2: 1 ; 0}^{2: 1: 0}\left[\begin{array}{l}
\left.\alpha+\frac{p}{2}+\frac{1}{2}, \beta+\frac{p}{2}+\frac{1}{2}:-m ;-\frac{y}{4},-\frac{y}{8}\right], \\
\frac{\alpha+\beta+p+1}{2}, \frac{\alpha+\beta+p+2}{2}: p+1 ; \ldots ;
\end{array}\right.
\end{aligned}
$$

where $\mathfrak{R}\left(\alpha+\frac{p}{2}\right)>-\frac{1}{2}, \mathfrak{R}\left(\beta+\frac{p}{2}\right)>-\frac{1}{2}$.

(ix) Further, on setting $k=\frac{1}{4}+p$ and $\mu=-\frac{1}{4}$ and using (16) in our first integral (8), we get

$$
\begin{aligned}
& \int_{0}^{1} x^{\alpha-1}(1-x)^{2 \beta-\frac{1}{2}}\left(1-\frac{x}{3}\right)^{2 \alpha-1}\left(1-\frac{x}{4}\right)^{\beta-\frac{3}{4}} \exp \left[\frac{-y\left(1-\frac{x}{4}\right)(1-x)^{2}}{2}\right] \\
& \times H_{2 p}\left[y\left(1-\frac{x}{4}\right)(1-x)^{2}\right]^{\frac{1}{2}} d x=\left(\frac{2}{3}\right)^{2 \alpha}(-1)^{-p} \frac{2 p !}{p !} \frac{\Gamma(\alpha) \Gamma\left(\beta+\frac{1}{4}\right)}{\Gamma\left(\alpha+\beta+\frac{1}{4}\right)} \\
& \times F_{1: 1: 0}^{1: 1: 0}\left[\begin{array}{c}
\beta+\frac{1}{4}: \quad-p ; \ldots ; \\
\alpha+\beta+\frac{1}{4}: \quad \frac{1}{2} ; \ldots ;
\end{array} \quad y,-\frac{y}{2}\right]
\end{aligned}
$$

where $\mathfrak{R}(\alpha)>0, \mathfrak{R}(\beta)>-\frac{1}{4}$. 
(x) Further, on setting $k=\frac{1}{4}+p$ and $\mu=-\frac{1}{4}$ and using (16) in our second integral (9), we get

$$
\begin{aligned}
& \int_{0}^{1} x^{\alpha-\frac{3}{4}}(1-x)^{2 \beta-\frac{1}{2}}\left(1-\frac{x}{3}\right)^{2 \alpha-1}\left(1-\frac{x}{4}\right)^{\beta-\frac{3}{4}} \exp \left[\frac{-x y\left(1-\frac{x}{4}\right)(1-x)^{2}}{2}\right] \\
& \times H_{2 p}\left[x y\left(1-\frac{x}{4}\right)(1-x)^{2}\right]^{\frac{1}{2}} d x=\left(\frac{2}{3}\right)^{2 \alpha}(-1)^{-p} \frac{2 p !}{p !} \frac{\Gamma\left(\alpha+\frac{1}{4}\right) \Gamma\left(\beta+\frac{1}{4}\right)}{\Gamma\left(\alpha+\beta+\frac{1}{2}\right)} \\
& \times F_{2: 1 ; 0}^{2: 1: 0}\left[\begin{array}{cccc}
\alpha+\frac{1}{4}, \quad \beta+\frac{1}{4}: & -p ; & -; & \frac{y}{4},-\frac{y}{8} \\
\frac{\alpha+\beta+\frac{1}{2}}{2}, & \frac{\alpha+\beta+\frac{3}{2}}{2}: & \frac{1}{2} ; \ldots ;
\end{array}\right] \text {, }
\end{aligned}
$$

where $\mathfrak{R}(\alpha)>-\frac{1}{4}, \mathfrak{R}(\beta)>-\frac{1}{4}$.

(xi) Further, on setting $k=\frac{3}{4}+p$ and $\mu=\frac{1}{4}$ and using (17) in our first integral (8), we get

$$
\begin{aligned}
& \int_{0}^{1} x^{\alpha-1}(1-x)^{2 \beta-\frac{1}{2}}\left(1-\frac{x}{3}\right)^{2 \alpha-1}\left(1-\frac{x}{4}\right)^{\beta-\frac{3}{4}} \exp \left[\frac{-y\left(1-\frac{x}{4}\right)(1-x)^{2}}{2}\right] \\
& \times H_{2 p+1}\left[y\left(1-\frac{x}{4}\right)(1-x)^{2}\right]^{\frac{1}{2}} d x=\left(\frac{1}{3}\right)^{2 \alpha} 2^{2 \alpha+1}(-1)^{-p} \frac{(2 p+1) !}{p !}(y)^{\frac{1}{2}} \frac{\Gamma(\alpha) \Gamma\left(\beta+\frac{3}{4}\right)}{\Gamma\left(\alpha+\beta+\frac{3}{4}\right)} \\
& \times F_{1: 1 ; 0}^{1: 1: 0}\left[\begin{array}{c}
\beta+\frac{3}{4}:-p ; \ldots ; \\
\alpha+\beta+\frac{3}{4}: \quad \frac{3}{2} ; \ldots ;
\end{array} \quad y,-\frac{y}{2}\right],
\end{aligned}
$$

where $\mathfrak{R}(\alpha)>0, \mathfrak{R}(\beta)>-\frac{3}{4}$.

(xii) Further, on setting $k=\frac{1}{4}+p$ and $\mu=-\frac{1}{4}$ and using (17) in our second integral (9), we get

$$
\begin{aligned}
& \int_{0}^{1} x^{\alpha-\frac{3}{4}}(1-x)^{2 \beta-\frac{1}{2}}\left(1-\frac{x}{3}\right)^{2 \alpha-1}\left(1-\frac{x}{4}\right)^{\beta-\frac{3}{4}} \exp \left[\frac{-x y\left(1-\frac{x}{4}\right)(1-x)^{2}}{2}\right] \\
& \times H_{2 p+1}\left[x y\left(1-\frac{x}{4}\right)(1-x)^{2}\right]^{\frac{1}{2}} d x=\left(\frac{1}{3}\right)^{2 \alpha} 2^{2 \alpha+1}(-1)^{-p} y^{\frac{1}{2}} \frac{(2 p+1) !}{p !} \frac{\Gamma\left(\alpha+\frac{3}{4}\right) \Gamma\left(\beta+\frac{3}{4}\right)}{\Gamma\left(\alpha+\beta+\frac{3}{2}\right)}
\end{aligned}
$$

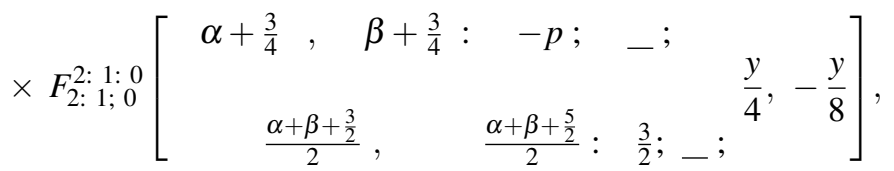

where $\mathfrak{R}(\alpha)>-\frac{3}{4}, \mathfrak{R}(\beta)-\frac{3}{4}$.

\section{Concluding Remark}

We investigate two unified integrals involving the Whittaker function of first kind, which are expressed in terms of Kampé de Fériet functions. Also, we discussed some special cases of our main results. The result obtained here are basic in nature and are likely to find useful applications in several fields notably electrical networks, probability theory and statistical mechanics. We are also trying to find certain possible applications of these results to some other research areas. 


\section{References}

[1] Ali, S. : On some new unified integrals, Adv. Comput. Math. Appl. 1(3) (2012), 151-153.

[2] Brychkov, Y. A. : Handbook of Special Functions: Derivatives, Integrals, Series and Other Formulas, CRC Press, Boca Raton (2008).

[3] Choi, J., Hasanov, A., Srivastava, H. M., and Turaev, M. : Integral representations for Srivastava's triple hypergeometric functions, Taiwan. J. Math. 15 (2011), 2751-2762.

[4] Choi, J. and Agarwal, P. : Certain unified integrals associated with Bessel functions, Boundary Value Problems, 2013(95) (2013), $1-9$.

[5] E. D. Rainville, Special functions, The Macmillan Company, New York, 1960.

[6] Garg, M. and Mittal, S. : On a new unified integral, Proc. Indian Acad. Sci. Math. Sci. 114(2) (2003), 99-101.

[7] J. L. Lavoie and G. Trottier, On the sum of certain Appell's series, Ganita, (1) 20 (1969), 31-32.

[8] Khan, N.U. and Ghayasuddin, M. Some unified integral associated with whittaker functions, Journal of Mathematical Analysis (Accepted).

[9] Khan, N.U. and Ghayasuddin, M. Study of unified integral associated with generalized Bessel-Maitland functions, Pure and Applied Mathematics Letters (Accepted).

[10] Khan, N.U. and Ghayasuddin, M. A new class of integral formulas associated with generalized Bessel functions, (Communicated).

[11] Srivastava, H. M and Manocha, H. L. : A Treatise on generating functions, Halsted Press (Ellis Horwood Limited, Chichester), John Wiley and Sons, New York, (1984).

[12] Whittaker, E. T. : An expression of certain known function as generalized hypergeometric functions, Bull. Amer. Math. Soc, 10 (1903), 125-134.

[13] Whittaker, E. T and Watson, G. N. : A course of modern analysis, 4th ed. Cambridge, England. Cambridge University, (1990). 